\title{
Heartbeat: Surgical risk scores in infective endocarditis
}

Infective endocarditis (IE) remains a highly mortal disease, with an in-hospital mortality of $15 \%-30 \%$, despite current imaging and microbiological diagnosis and appropriate antibiotic therapy. One of the challenges in improving outcomes in patients with IE is risk stratification and optimal timing of surgical intervention. In this issue of Heart, Olmos and colleagues ${ }^{1}$ retrospectively analysed outcomes in 671 patients who underwent cardiac surgery for left-sided IE to develop and validate a risk score for prediction of perioperative mortality. This Risk-Endocarditis (RISK-E) score is based on both IE-specific factors, such as microorganism, prosthetic infection, paravalvular involvement and sepsis, and surgical factors, such as age, haemodynamics, thrombocytopenia and renal failure (table 1 and figure 1 ).

In the accompanying editorial, Donal, Flecher and Tattevin ${ }^{2}$ discuss the limitations of this proposed risk score and emphasise the importance of the Endocarditis Team in making treatment decisions. "Although not all patients with IE will require cardiac surgery during the acute phase, this will eventually be the case for approximately half of them, and the endocarditis team should be rapidly contacted for the decision-making process. Risk stratification plays an important role for the selection of cases that will benefit from cardiac surgery, and the reasoning is based on this risk-benefit balance, that is, what is the risk associated with an early surgical approach compared with a conservative management."

Recent studies support the important of measuring stroke volume index (SVI) in adults with aortic stenosis (AS) who have a small valve area despite only a moderate transvalvular velocity or gradient. However, whether SVI is predictive of long-term outcomes in patients with initially mild or moderate AS has not been previously studied. Lønnebakken and colleagues ${ }^{3}$ examined the association between SVI and major cardiovascular (CV) events over a median 4.3 years of follow-up for 1671 adults with moderate AS enrolled in the Simvastatin Ezetimibe in Aortic Stenosis study. As seen in figure 2,

Correspondence to Professor Catherine M Otto Division of Cardiology, University of Washington, Seattle, Washington, USA; cmotto@uw.edu

\begin{tabular}{|c|c|c|}
\hline & Score & Points \\
\hline \multicolumn{3}{|l|}{ Age (years) } \\
\hline 51 & 0 & \\
\hline $52-63$ & 9 & 9 \\
\hline $64-72$ & 13 & \\
\hline$\geq 73$ & 14 & \\
\hline Prosthetic endocarditis & 6 & 6 \\
\hline Virulent microorganism* & 9 & 9 \\
\hline Septic shock & 7 & \\
\hline Thrombocytopeniat & 7 & \\
\hline Acute renal insufficiency & 5 & 5 \\
\hline Cardiogenic shock & 15 & \\
\hline Periannular complications & 5 & \\
\hline RISK-E score & 29 & 29 \\
\hline
\end{tabular}

This table illustrates an example of the RISK-E calculation for a 60-year-old man with prosthetic valve endocarditis due to Staphylococcus aureus and acute renal insufficiency. The points of the score present in this case are represented in bold characters. The total RISK-E was 29 points, and the predicted mortality was 39.6\%.

* Staphylococcus aureus or fungi.

†Thrombocytopenia (<150000 platelet $\left./ \mathrm{mm}^{3}\right)$.

$\ddagger$ Presence of abscess, pseudoaneurysm, fistula or prosthetic dehiscence.

a low SVI $\left(<35 \mathrm{~mL} / \mathrm{m}^{2}\right.$, present in $23 \%$ of subjects at baseline) was associated with higher total mortality and CV events with an HR for major CV events of 1.09 (95\% CI 1.05 to $1.13, \mathrm{p}<0.001)$ and an HR of

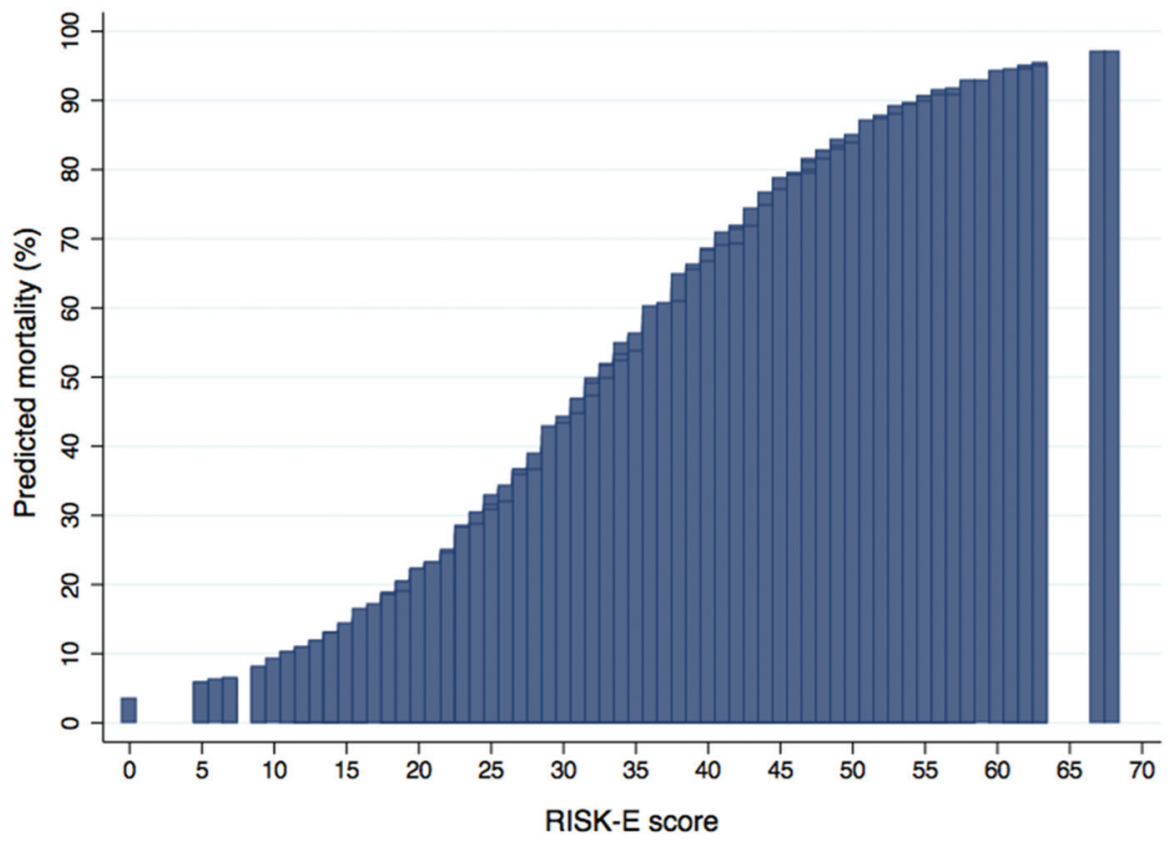

Figure 1 Predicted risk of postoperative mortality associated with individual Risk-Endocarditis (RISK-E) scores, according to the presence and scoring of each risk factor. $1.16, \mathrm{p}=0.038$ ) for a $5 \mathrm{~mL} / \mathrm{m}^{2}$ lower SVI at baseline.

Singh and $\mathrm{McCann}^{4}$ review the approach to measurement of SVI by
1.08 for total mortality $(95 \%$ CI 1.01 to 


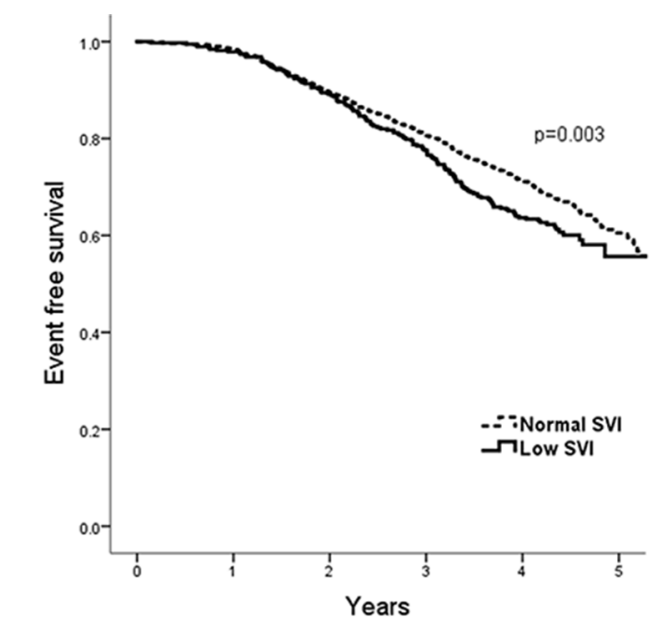

$\begin{array}{lrrrrrr}\text { Low SVI } & 379 & 370 & 331 & 282 & 126 & 8 \\ \text { Normal SVI } & 1292 & 1282 & 1157 & 1032 & 896 & 69\end{array}$

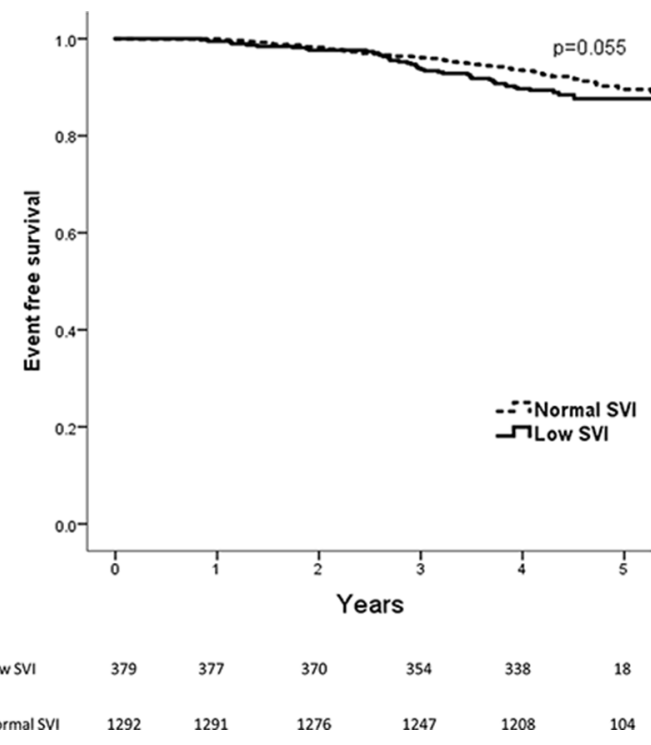

Figure 2 Kaplan-Meier plot demonstrating event-free survival in patients with low and normal (stroke volume index (SVI)), respectively.

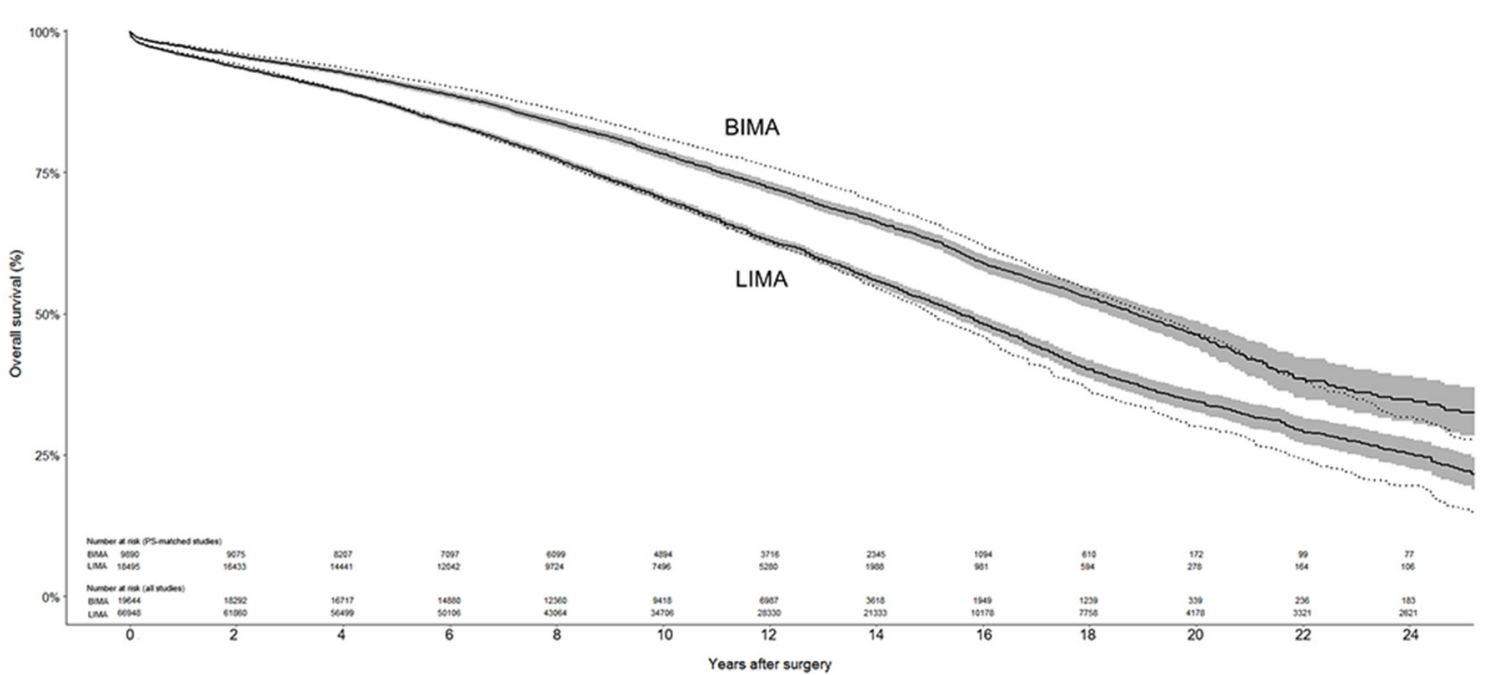

Figure 3 Reconstructed overall survival for bilateral internal mammary artery (BIMA) versus single (left) internal mammary artery (LIMA) use in coronary artery grafting. Solid line represents aggregated survival curves of propensity-matched or risk-adjusted patient cohorts, with the grey region indicating $95 \% \mathrm{Cl}$. Dotted line represents aggregation of all available Kaplan-Meier curves, irrespective of matching or risk adjustment.

Doppler echocardiography and potential inaccuracies due to underestimation of outflow tract cross-sectional area. In addition, "SVI is dependent on multiple factors, including LV volume, contractility, filling pressures and afterload, and not just valvular obstruction, and therefore will be influenced by other common comorbidities such as coronary artery disease and hypertension." Given the only modest association of SVI with outcomes and the importance of symptom status in clinical decision-making, they suggest caution in using SVI in evaluation of mild-to-moderate AS in routine clinical practice.

The use of bilateral internal mammary arteries (BIMA) for coronary revascularisation improves long-term survival compared with single/left internal mammary artery (LIMA) grafting. However, the relative risks and benefits of other outcomes with these two procedures are less clear. This question was addressed in a meta-analysis of 29 observational studies including over 89000 patients by Buttar and colleagues. ${ }^{5}$ In both unmatched and propensity-matched cohorts, survival was higher with BIMA compared with LIMA (HR 0.78, $\mathrm{p}<0.00001) \quad$ Both in-hospital and late mortality (figure 3) were lower with BIMA compared with LIMA. In addition, BIMA was associated with a lower risk of cerebrovascular accidents $(1.3 \%$ vs $2.9 \%, \mathrm{p}=0.0003)$ and need for revascularisation $(4.8 \%$ vs $10 \%$, $\mathrm{p}=0.005)$. However, deep sternal wound infection occurred more often with BIMA compared with LIMA $(1.8 \%$ vs $1.4 \%$, $\mathrm{p}=0.0008)$.

Kurlansky ${ }^{6}$ wisely comments, "The art of medicine might well be described as the ability to make the right decisions based on incomplete information. Try as we might to provide 'evidence-based' care, the more scientifically candid among us will confess that the 'definitive study' on any given topic never has been (nor ever will be) performed." He goes on to discuss the difficulties in performing a meaningful randomised controlled clinical trial comparing LIMA to BIMA and the limitation of observational studies, even when combined in a large meta-analysis. Even so, he concludes, "This surgeon, should 


\section{a) Confounding}

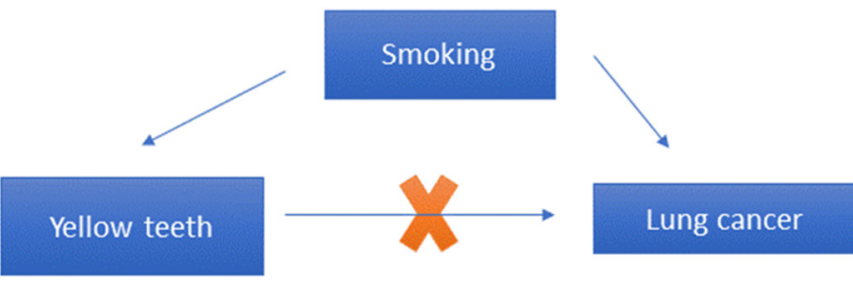

b) Reverse causality

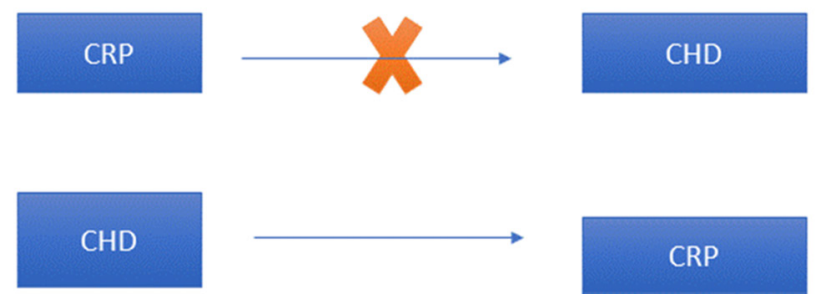

Figure 4 Examples of $(A)$ confounding and (B) reverse causality in observational epidemiology. (A) The arrows denote the direction of proposed causality and the cross denotes that the postulated direct link between yellow teeth and lung cancer is false. (B) The arrows denote the direction of proposed causality and the cross denotes that the postulated direct link between $C$ reactive protein (CRP) and coronary heart disease (CHD) is false and in fact the current evidence suggests that CHD raises levels of CRP (ie, the arrow goes in the opposite direction).

\section{Conventional Trial}

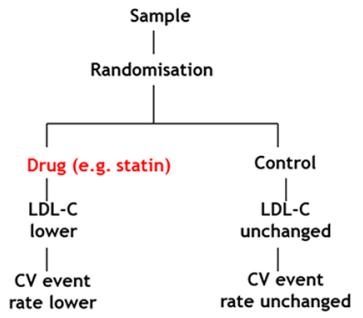

\section{Mendelian randomisation}

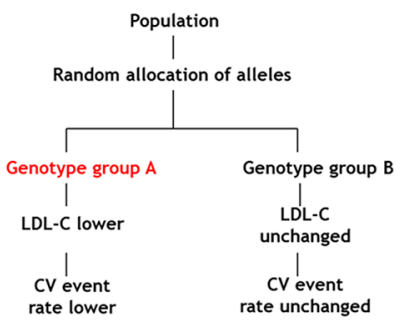

Figure 5 Comparison of a conventional trial with a Mendelian randomisation study. This illustrates the analogy between a conventional randomised controlled trial and a Mendelian randomisation study. CV, cardiovascular, low density lipoprotein cholesterol (LDL-C).

he need coronary revascularisation today, would chose a surgeon accomplished in the art of BIMA grafting."

An excellent review article in this issue explains the concepts of Mendelian randomisation for CV research. ${ }^{7}$ This approach can provide key data to identify new drug targets, as well as predicting efficacy and adverse effects (figures 4 and 5) "There are three key rules for the conduct of a valid Mendelian randomisation study: (1) that the genetic variant associates with the biomarker, (2) the genetic variant is not associated with confounders of the biomarker to outcome association and (3) that the genetic variant only influences risk of disease through the biomarker of interest. Violation of any of these three rules can lead to a biased estimate, meaning that the causal estimate may not be reliable."

\section{Competing interests None declared.}

Provenance and peer review Commissioned; internally peer reviewed.

(c) Article author(s) (or their employer(s) unless otherwise stated in the text of the article) 2017. All rights reserved. No commercial use is permitted unless otherwise expressly granted.

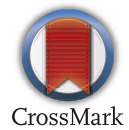

To cite Otto CM. Heart 2017;103:1391-1393.

Heart 2017;103:1391-1393.

doi:10.1136/heartjnl-2017-312238

\section{REFERENCES}

1 Olmos C, Vilacosta I, Habib G, et al. Risk score for cardiac surgery in active left-sided infective endocarditis. Heart 2017;103:1435-42.

2 Donal E, Flecher E, Tattevin P. Machine learning to support decision-making for cardiac surgery during the acute phase of infective endocarditis. Heart 2017;103:1396-7.

3 Lønnebakken MT, De Simone G, Saeed S, et al. Impact of stroke volume on cardiovascular risk during progression of aortic valve stenosis. Heart 2017;103:1443-8.

4 Singh A, McCann GP. Stroke volume index in mildmoderate aortic stenosis: more than a barometer of systolic function? Heart 2017;103:1398-9.

5 Buttar SN, Yan TD, Taggart DP, et al. Long-term and short-term outcomes of using bilateral internal mammary artery grafting versus left internal mammary artery grafting: a meta-analysis. Heart 2017;103:1419-26.

6 Kurlansky PA. Arterial grafting for coronary revascularisation and the illusive search for truth. Heart 2017;103:1394-5.

7 Bennett DA, Holmes MV. Mendelian randomisation in cardiovascular research: an introduction for clinicians. Heart 2017;103:1400-7. 